\title{
Precise RTK Positioning with GPS/ INS Tight Coupling and Multipath Estimation
}

\author{
Patrick Henkel ${ }^{*, * *}$ and Andreas Sperl ${ }^{*, * *}$ \\ *Advanced Navigation Solutions - ANavS, Munich, Germany \\ **Technische Universität München (TUM), Munich, Germany
}

\section{BIOGRAPHIES}

Patrick Henkel received his Bachelor, Master and $\mathrm{PhD}$ degrees from the Technische Universität München, Munich, Germany. In 2010, he graduated with a PhD thesis on reliable carrier phase positioning (summa cum laude) and is now working towards his habilitation in the field of precise point positioning. He visited the Mathematical Geodesy and Positioning group at TU Delft in 2007, and the GPS Lab at Stanford University in 2008 and 2010. Dr. Henkel received the Pierre Contensou Gold Medal in 2007, the 1st prize in Bavaria at the European Satellite Navigation Competition in 2010, and the Vodafone Award for his dissertation in 2011. He is one of the founders and currently also the managing director of Advanced Navigation Solutions - ANavS.

Andreas Sperl studied Electrical and Computer Engineering at the Technische Hochschule Deggendorf and the Technische Universität München, Munich, Germany. His master thesis was entitled "Joint RTK and Attitude determination" and evaluated with the grade "with distinction". Mr. Sperl also did an apprenticeship for Electronic Technicians and has an extensive experience in the design of printed circuit boards. He is is working as a hardware and software developer on RTK positioning at ANavS since 2015.

\footnotetext{
ABSTRACT

Real-Time Kinematic (RTK) positioning is attractive for numerous applications including autonomous driving of vehicles. However, multipath remains a challenge for RTK positioning especially in urban environments. Choke-ring antennas which suppress code multipath cannot be used due to restrictions on the size, weight and costs. The used low-cost patch antennas cannot suppress the multipath, which can be of several tens of meters.

Therefore, we will include a code multipath parameter for each satellite in our RTK positioning to prevent a map-
}

ping of the multipath into other state parameters and to exploit the temporal correlation of the multipath. We also enhance the ambiguity fixing by introducing two fixing phases: A candidate determination phase and a candidate tracking phase. In the first phase, we determine sets of integer candidate vectors using LAMBDAs integer decorrelation and tree search [1]. As the float solution and/ or its statistics might be biased, we determine integer candidate vectors at multiple epochs with different float solutions and merge these integer candidate vectors. Thereby, we increase the likelihood of including the correct candidate vector in the set of candidate vectors. In the second phase, a conditional least-squares phase-only baseline estimate is determined for each candidate vector at every epoch. The sum of squared measurement residuals is accumulated over time for every candidate vector. This second phase has two important advantages over an instantaneous decision: First, the accumulation of the residuals improves the discrimination between candidates. Secondly, the used single epoch least-squares phase-only solutions are not affected by the float solution. This is helpful since any temporal correlation in the phase measurements (e.g. due to phase multipath) could lead to erroneous statistics of the float solution. We fix the RTK and attitude ambiguities sequentially: First, the attitude ambiguities are fixed in a tree search using soft a priori information on the baseline length. Subsequently, the RTK ambiguities are fixed in another tree search using both the measurements of the RTK baseline and the fixed measurements of the attitude baseline. We select the final candidate based on the accumulated sum of squared phase residuals and the baseline stability.

The fixed attitude enables a precise estimation of the accelerometer and angular rate biases. A precise RTK position and attitude is then be obtained by tracking the fixed solution with GPS/ INS tight coupling. We show the RTK performance for both static and kinematic measurements: We obtained a millimeter-level positioning accuracy for static conditions and a centimeter-level positioning accuracy for kinematic conditions with multipath errors of up to $50 \mathrm{~m}$. 


\section{INTRODUCTION}

The estimation of a code multipath parameter for each satellite has several advantages. The following list includes the most important benefits of multipath estimation.

\section{Benefits of code multipath estimation for RTK:}

- Separation of multipath errors from baseline and ambiguity estimates, i.e. unbiased estimation of float ambiguities, baseline and multipath

- Reduction of variance of code measurement noise to variance of pure noise

- Exploitation of temporal correlation of multipath

- Faster convergence of float ambiguities

- Increase of probability of including correct integer ambiguities in integer search space

- Increase of probability of selecting correct integer ambiguities

\section{Further consequences of code multipath estimation:}

- measurements from multiple epochs are needed to estimate baseline, ambiguities and a multipath parameter for each satellite

- separation of baseline from ambiguities and multipath is enabled only through temporal changes of satellitereceiver line of sight vectors $\vec{e}_{r}^{k}$

\section{MEASUREMENT SET-UP}

In this paper, we consider the measurement set-up of Fig. 1 [2]. Two single-frequency GPS patch antennas are mounted on top of a vehicle and connected to two u-blox LEA 6T GPS receivers to get the two-dimensional attitude (heading, pitch) of the vehicle. A virtual reference station (VRS, see Landau et al. [3]) serves as a third receiver for RTK positioning. An additional low-cost inertial sensor (MPU 9150 from Invensense) provides 3D acceleration and angular rate measurements. The inertial sensor allows a reliable cycle slip correction and position/ attitude determination during short GNSS signal interruptions.

We consider the measurements of all three receivers and the inertial sensor jointly in a Kalman filter [4]. The individual sensors have different data rates, i.e. $1 \mathrm{~Hz}$ for the VRS, $5 \mathrm{~Hz}$ for the GPS receivers and $100 \mathrm{~Hz}$ for the inertial sensor. The position and attitude estimate is updated whenever there is a GPS or inertial measurement available. This yields an update rate of $105 \mathrm{~Hz}$ for the position and attitude.

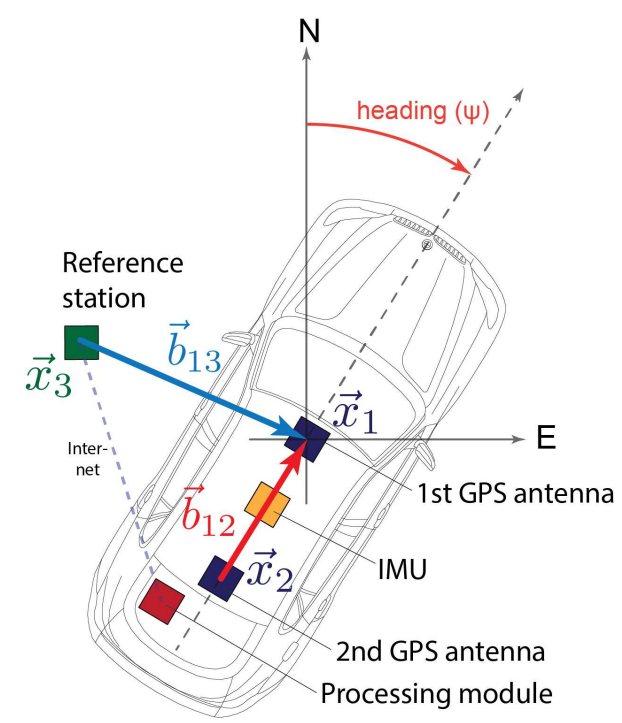

Fig. 1: Measurement set-up: Two GPS antennas on the vehicle are used for attitude determination. The (virtual) reference station allows an RTK positioning. The inertial measurement unit (IMU) is used for cycle slip correction and position/ attitude determination during short GPS signal interruptions.

\section{RTK POSITIONING WITH MULTIPATH ESTIMATION}

Our RTK positioning includes the following processing steps:

- float attitude solution: estimation of attitude baseline, ambiguities and $D D$ code multipaths with a Kalman filter using DD pseudorange and carrier phase measurements

- float RTK solution: estimation of RTK baseline, ambiguities and DD code multipaths with a Kalman filter using DD pseudorange and carrier phase measurements

- fixing of attitude DD ambiguities

- conditional fixing of RTK DD ambiguities using DD pseudorange and carrier phase measurements from both attitude and RTK baselines and fixed attitude ambiguities

- calibration of accelerometer and gyroscope, i.e. estimation of sensor biases using GPS-based attitude

- estimation of position and attitude with GPS/ INS tight coupling and fixed attitude and RTK ambiguities

In the tight coupling, we use satellite-satellite single difference (SD) measurements instead of double difference (DD) measurements. The use of SD instead of DD measurements has one major advantage: As the data rate of the 


$$
\begin{aligned}
& \left(\begin{array}{c}
\lambda \tilde{\varphi}_{1}^{k l} \\
\lambda \tilde{\varphi}_{2}^{k l} \\
\lambda \tilde{\varphi}_{3}^{k l} \\
\hline \tilde{\rho}_{1}^{k l} \\
\tilde{\rho}_{2}^{k l} \\
\tilde{\rho}_{3}^{k l} \\
\hline \tilde{f}_{\mathrm{D}_{1}}^{k l} \\
\tilde{f}_{\mathrm{D}_{2}}^{k l}
\end{array}\right)=\left(\begin{array}{c}
\lambda \varphi_{1}^{k l} \\
\lambda \varphi_{2}^{k l} \\
\lambda \varphi_{3}^{k l} \\
\hline \rho_{1}^{k l} \\
\rho_{2}^{k l} \\
\rho_{3}^{k l} \\
\hline f_{\mathrm{D}_{1}^{k l}}^{k l} \\
f_{\mathrm{D}_{1}}^{k l}
\end{array}\right)-\left(\begin{array}{lll}
\vec{e}_{1}^{k l} \vec{x}_{3}+\vec{e}_{1}^{k} \vec{x}^{k}-\vec{e}_{1}^{l} \vec{x}^{l}+c \delta t^{k l}-T_{1}^{k l} & -\lambda\left(N_{13}^{k l}\right) & -\lambda / 2\left(\Delta N_{13}^{k l}\right) \\
\vec{e}_{1}^{k l} \vec{x}_{3}+\vec{e}_{1}^{k} \vec{x}^{k}-\vec{e}_{1}^{l} \vec{x}^{l}+c \delta t^{k l}-T_{1}^{k l}+c_{12}^{k l}-\lambda\left(N_{13}^{k l}-N_{12}^{k l}\right) & -\lambda / 2\left(\Delta N_{13}^{k l}-\Delta N_{12}^{k l}\right) \\
\vec{e}_{1}^{k l} \vec{x}_{3}+\vec{e}_{1}^{k} \vec{x}^{k}-\vec{e}_{1}^{l} \vec{x}^{l}+c \delta t^{k l}-T_{1}^{k l}+c_{13}^{k l} & \\
\hline \vec{e}_{1}^{k l} \vec{x}_{3}+\vec{e}_{1}^{k} \vec{x}^{k}-\vec{e}_{1}^{l} \vec{x}^{l}+c \delta t^{k l}-T_{1}^{k l} \\
\vec{e}_{1}^{k l} \vec{x}_{3}+\vec{e}_{1}^{k} \vec{x}^{k}-\vec{e}_{1}^{l} \vec{x}^{l}+c \delta t^{k l}-T_{1}^{k l}+c_{12}^{k l} & \\
\vec{e}_{1}^{k l} \vec{x}_{3}+\vec{e}_{1}^{k} \vec{x}^{k}-\vec{e}_{1}^{l} \vec{x}^{l}+c \delta t^{k l}-T_{1}^{k l}+c_{13}^{k l} & \\
\hline f_{\mathrm{c}}\left(\vec{e}_{1}^{k} \vec{v}^{k}-\vec{e}_{1}^{l} \vec{v}^{l}\right) / c+f_{\mathrm{c}} \delta \dot{t}^{k l} \\
f_{\mathrm{c}}\left(\vec{e}_{1}^{k l} \vec{v}^{k}-\vec{e}_{1}^{l} \vec{v}^{l}\right) / c+f_{\mathrm{c}} \delta \dot{t}^{k l}
\end{array}\right) \\
& =\left(\begin{array}{lrr}
\vec{e}_{1}^{k l} \vec{b}_{13} & +\lambda N_{3}^{k l}-I^{k l}+\lambda \beta^{k l} & +\varepsilon_{1}^{k l} \\
\vec{e}_{1}^{k l}\left(\vec{b}_{13}-\vec{b}_{12}\right) & +\lambda N_{3}^{k l}-I^{k l}+\lambda \beta^{k l} & +\varepsilon_{2}^{k l} \\
& +\lambda N_{3}^{k l}-I^{k l}+\lambda \beta^{k l} & +\varepsilon_{3}^{k l} \\
\hline \vec{e}_{1}^{k l} \vec{b}_{13} & +\Delta \rho_{\mathrm{MP}_{1}}^{k l}+I^{k l}+b^{k l} & +\eta_{1}^{k l} \\
\vec{e}_{1}^{k l}\left(\vec{b}_{13}-\vec{b}_{12}\right) & +\Delta \rho_{\mathrm{MP}_{2}}^{k l}+I^{k l}+b^{k l} & +\eta_{2}^{k l} \\
& +\Delta \rho_{\mathrm{MP}}^{k l}+I^{k l}+b^{k l} & +\eta_{3}^{k l} \\
\hline-f_{\mathrm{c}} \vec{e}_{1}^{k l} \overrightarrow{\vec{b}}_{13} / c & & \eta_{f_{\mathrm{D}_{1}}}^{k l} \\
-f_{\mathrm{c}} \vec{e}_{1}^{k l}\left(\dot{\vec{b}}_{13}-\dot{\vec{b}}_{12}\right) / c & \eta_{f_{\mathrm{D}_{2}}}^{k l}
\end{array}\right)
\end{aligned}
$$

VRS is much lower than the data rate of the GPS receivers, DD measurements can only be computed at the low rate of the VRS while SD measurements can be determined with the individual, partially higher data rates.

We correct the SD measurements for the known position of the VRS, the known satellite positions and clock offsets, the tropospheric delays, the synchronization corrections, the fixed DD ambiguities and cycle slips. This leaves the RTK and attitude baselines, the SD ambiguities $N_{3}^{k l}$ of the VRS, the SD slant ionospheric delays $I^{k l}$, the SD satellite phase biases $\beta^{k l}$, the SD code multipath parameters $\Delta \rho_{\mathrm{MP} r}^{k l}$ of each receiver $r \in\{1,2,3\}$ and the SD satellite code biases $b^{k l}$ as unknowns. We additionally consider the SD Doppler measurements of the two GPS receivers, which provide an information on the receiver velocity (i.e. rate of the RTK baseline) and the rate of attitude baseline. Thus, we obtain the model of Eq. (1) for the GPS measurements as decribed by Sperl in [5].

As the SD ambiguities of the VRS, the slant ionospheric delays and the SD satellite phase biases can not be separated for single-frequency GPS receivers, we will treat them together as a lumped real-valued term. Similarly, we will map the slant ionospheric delays and satellite code biases to the SD multipath parameter.

The MEMS-based inertial sensors are providing the measurements in a local body-fixed frame (denoted by the upper index $\mathrm{b}$ ). We relate the acceleration in the b-frame to the acceleration in the Earth-Centered Earth Fixed (ECEF) frame according to Jekeli [6] and Henkel [7] as

$$
\begin{aligned}
a^{\mathrm{b}}\left(t_{n}\right)= & R_{\mathrm{n}}^{\mathrm{b}}\left(t_{n}\right) R_{\mathrm{e}}^{\mathrm{n}}\left(t_{n}\right) a^{\mathrm{e}}\left(t_{n}\right)+b_{a}^{\mathrm{b}}\left(t_{n}\right) \\
& +g\left(\begin{array}{c}
\sin \left(\theta\left(t_{n}\right)\right) \\
\cos \left(\theta\left(t_{n}\right)\right) \sin \left(\phi\left(t_{n}\right)\right) \\
\cos \left(\theta\left(t_{n}\right)\right) \cos \left(\phi\left(t_{n}\right)\right)
\end{array}\right)+\varepsilon_{a}^{\mathrm{b}}\left(t_{n}\right)
\end{aligned}
$$

with the rotation matrices $R_{\mathrm{e}}^{\mathrm{n}}$ and $R_{\mathrm{n}}^{\mathrm{b}}$, the acceleration $a^{\mathrm{e}}$ in the e-frame being equal to $\ddot{\vec{b}}_{13}$, the acceleration biases $b_{a}^{\mathrm{b}}$ of the sensor in the b-frame, the gravitational acceleration $g$, the pitch angle $\theta$, the roll angle $\phi$ and the measurement noise $\varepsilon_{a}^{\mathrm{b}}$. The rotation from the e-frame into the $\mathrm{n}$-frame depends on the latitude $\varphi_{1}$ and longitude $\lambda_{1}$ of receiver 1 and is given by

$$
R_{\mathrm{e}}^{\mathrm{n}}\left(t_{n}\right)=R_{1}\left(\pi / 2-\varphi_{1}\left(t_{n}\right)\right) R_{3}\left(\pi / 2+\lambda_{1}\left(t_{n}\right)\right) .
$$

The rotation from the $\mathrm{n}$-frame into the $\mathrm{b}$-frame depends on the heading $\psi$ and pitch $\theta$ of the vehicle and is given by

$$
R_{\mathrm{n}}^{\mathrm{b}}\left(t_{n}\right)=R_{2}\left(-\theta\left(t_{n}\right)\right) R_{3}\left(\pi / 2-\psi\left(t_{n}\right)\right) .
$$

The gyroscope senses the angular rates $\omega_{\mathrm{ib}}^{\mathrm{b}}$ of the bodyfixed (b-) frame w.r.t. the inertial (i-) frame in the b-frame. The angular rate measurements can be expressed as the sum of $\omega_{\mathrm{in}}^{\mathrm{b}}, \omega_{\mathrm{nb}}^{\mathrm{b}}$, a bias $b_{\omega_{\mathrm{ib}}}^{\mathrm{b}}$ and a noise $\eta_{\omega_{\mathrm{ib}}}^{\mathrm{b}}$, i.e.

$$
\omega_{\mathrm{ib}}^{\mathrm{b}}\left(t_{n}\right)=R_{\mathrm{n}}^{\mathrm{b}}\left(t_{n}\right) \omega_{\mathrm{in}}^{\mathrm{n}}\left(t_{n}\right)+\omega_{\mathrm{nb}}^{\mathrm{b}}\left(t_{n}\right)+b_{\omega_{\mathrm{ib}}}^{\mathrm{b}}\left(t_{n}\right)+\eta_{\omega_{\mathrm{ib}}}^{\mathrm{b}}\left(t_{n}\right)
$$

The angular rates $\omega_{\mathrm{nb}}^{\mathrm{b}}$ are related to the rates of the Euler angles according to Jekeli [6] as

$$
\begin{aligned}
\omega_{\mathrm{nb}}^{\mathrm{b}} & =R_{1}(\phi) R_{2}(\theta)\left(\begin{array}{c}
0 \\
0 \\
\dot{\psi}
\end{array}\right)+R_{1}(\phi)\left(\begin{array}{c}
0 \\
\dot{\theta} \\
0
\end{array}\right)+\left(\begin{array}{c}
\dot{\phi} \\
0 \\
0
\end{array}\right) \\
& =\left(\begin{array}{ccc}
1 & 0 & -\sin (\theta) \\
0 & \cos (\phi) & \cos (\theta) \sin (\phi) \\
0 & -\sin (\phi) & \cos (\theta) \cos (\phi)
\end{array}\right)\left(\begin{array}{c}
\dot{\phi} \\
\dot{\theta} \\
\dot{\psi}
\end{array}\right),(6)
\end{aligned}
$$

with $R_{i}(\alpha)$ being a rotation around the $i$-th axis by an angle $\alpha$. The rotation $\omega_{\text {in }}^{\mathrm{n}}$ of the navigation frame w.r.t. the inertial frame depends on the latitude $\varphi_{1}$, the rates $\left\{\dot{\varphi}_{1}, \dot{\lambda}_{1}\right\}$ of 
latitude and longitude, and the Earth rotation rate $\omega_{\mathrm{e}}$, and is given by Jekeli [6] as

$$
\omega_{\mathrm{in}}^{\mathrm{n}}=\left(\begin{array}{c}
\left(\dot{\lambda}_{1}+\omega_{\mathrm{e}}\right) \cos \left(\varphi_{1}\right) \\
-\dot{\varphi}_{1} \\
-\left(\dot{\lambda}_{1}+\omega_{\mathrm{e}}\right) \sin \left(\varphi_{1}\right),
\end{array}\right) .
$$

In the tight coupling, we estimate the following state parameters (see also [8]):

$$
\begin{aligned}
x= & \left(\vec{b}_{13}^{\mathrm{T}}, \dot{\vec{b}}_{13}^{\mathrm{T}}, \ddot{\vec{b}}_{13}^{\mathrm{T}}, \psi, \theta, \phi, \dot{\psi}, \dot{\theta}, \dot{\phi},\left(\lambda N_{3}-I+\lambda \beta\right)^{\mathrm{T}}, \ldots\right. \\
& \left(\Delta \rho_{\mathrm{MP}_{1}}+I+b\right)^{\mathrm{T}},\left(\Delta \rho_{\mathrm{MP}_{2}}+I+b\right)^{\mathrm{T}} \\
& \left.\left(\Delta \rho_{\mathrm{MP}_{3}}+I+b\right)^{\mathrm{T}}, b_{a}^{\mathrm{b}}, b_{\omega_{\mathrm{ib}}}^{\mathrm{b}}\right)^{\mathrm{T}}
\end{aligned}
$$

Note that this state vector differs from the classical tight coupling by the additional SD ambiguities of the VRS and the SD code multipath parameters of each receiver.

\section{MODELING OF CODE MULTIPATH}

We model the pseudorange multipath at epoch $n$ as a scaled Random-walk process, i.e.

$$
\Delta \rho_{\mathrm{MP}_{r, n}}^{k}=\alpha_{n} \Delta \rho_{\mathrm{MP}_{r, n-1}}^{k}+\left(1-\alpha_{n}\right) \eta_{\Delta \rho_{\mathrm{MP}_{r, n}}^{k}}
$$

where the scaling factor $\alpha_{n} \in[0,1]$ was introduced for the following reason:

- modeling of both static multipath ( $\alpha_{n}$ equal to 1 results in deterministic multipath) and kinematic multipath ( $\alpha_{n}$ equal to 0 corresponds to white noise)

- variance of multipath is bounded for $n \rightarrow \infty$ if $\alpha_{n}<1$

Consequently, the parameter $\alpha_{n}$ should be a function of the speed of the object, i.e. the value should be reduced for increasing speed.

The code multipath at epoch $n$ can be further developed with Eq. (9) as:

$$
\begin{aligned}
\Delta \rho_{\mathrm{MP}_{r, n}}^{k}= & \left(\prod_{i=1}^{n} \alpha_{i}\right) \Delta \rho_{\mathrm{MP}_{r, 0}}^{k} \\
& +\sum_{i=0}^{n-1}\left(\prod_{j=n-i+1}^{n} \alpha_{j}\right)\left(1-\alpha_{n-i}\right) \eta_{\Delta \rho_{\mathrm{MP}_{r, n-i}}^{k}}
\end{aligned}
$$

We model the change of the multipath over time as white Gaussian noise, i.e.

$$
\eta_{\Delta \rho_{\mathrm{MP} r, n}^{k}} \sim \mathcal{N}\left(0, \sigma_{\Delta \rho_{\mathrm{MP}_{r, n}}^{k}}^{2}\right) .
$$

Thus, the variance of the code multipath follows as

$$
\begin{aligned}
\sigma_{\Delta \rho_{\mathrm{MP}_{r, n}}^{2}}^{2} & \left(\prod_{i=1}^{n} \alpha_{i}^{2}\right) \sigma_{\Delta \rho_{\mathrm{MP}_{r, 0}}^{2}}^{2} \\
& +\sum_{i=0}^{n-1}\left(\prod_{j=n-i+1}^{n} \alpha_{j}^{2}\right)\left(1-\alpha_{n-i}\right)^{2} \sigma_{\eta_{\Delta \rho_{\mathrm{MP}}, n-i}}^{2} .
\end{aligned}
$$

The variances can be further simplified in case of a constant $\alpha_{n}$ and constant $\sigma_{\eta_{\Delta \rho_{\mathrm{MP}}^{k}, n-i}}^{2}$. We use a finite geometric series expansion to simplify Eq. (12) to

$$
\sigma_{\Delta \rho_{\mathrm{MP}_{r, n}}^{k}}^{2}=\alpha^{2 n} \sigma_{\Delta \rho_{\mathrm{MP}_{r, 0}}^{k}}^{2}+(1-\alpha)^{2} \frac{1-\alpha^{2 n}}{1-\alpha^{2}} \sigma_{\Delta \rho_{\mathrm{MP} r, n}^{k}}^{2}
$$

For large $n$, the variances converge to the finite value

$$
\lim _{n \rightarrow \infty} \sigma_{\Delta \rho_{\mathrm{MP}_{r, n}}^{k}}^{2}=\frac{(1-\alpha)^{2}}{1-\alpha^{2}} \sigma_{\Delta \rho_{\mathrm{MP}_{r}}^{k}}^{2} .
$$

\section{ENHANCED RTK POSITIONING}

In this section, we describe our enhancements to the classical RTK positioning.

- Float solution:

In the state of the art RTK positioning [3], the 3D baseline and DD ambiguities are estimated from DD phase and code measurements. The code multipath is not considered as a parameter to be estimated. It is implicitly assumed that it can be mapped to the code noise, and the respective variance is increased accordingly.

Unfortunately, this assumption is not valid as the code multipath is having a substantial deterministic part (especially for static situations), which can not be reduced by averaging over multiple epochs and results in a significant temporal correlation. If the code multipath exceeds the assumed code noise variance, a certain part of the multipath is also mapped to the ambiguities and baseline estimates, which results in a heavily biased float solution.

However, the temporal correlation of multipath could be fully exploited in a state space model. We include a multipath parameter for each DD in the float solution to obtain the following advantages:

- prevent mapping of multipath errors to ambiguities and baseline

- exploit temporal correlation of multipath

- better use of code measurements by setting code noise variance to variance of pure noise 
- Triggering of fixing:

The well-known LAMBDA method of Teunissen [1] uses the float ambiguity covariance matrix for ambiguity fixing. The covariance matrix of the float ambiguity solution might be erroneous for the following reasons:

- covariance matrix of DD phase and code measurements is not accurately known, i.e. the correlation between the DD measurements is not properly modeled or estimated

- the phase multipath and its temporal correlation are not taken into account

- receiver tracking errors are not accurately considered in the measurement model

As these errors often lead to an underestimation of the actual uncertainty, we use the stability of the float solution as additional criterion to trigger the fixing. The stability is determined from the maximum variation of the float baseline estimate $\hat{\vec{b}}_{12}^{(i)}$, whereas the maximization is taken over the last $j_{\mathrm{s}}=\left\{0, \ldots, j_{\max }\right\}$ epochs and over the three coordinates $i \in\{1,2,3\}$, i.e.

$$
\max _{i \in\{1,2,3\}}\left(\max _{j \in j_{\mathrm{s}}} \hat{\vec{b}}_{12}^{(i)}\left(t_{n-j}\right)-\min _{j \in j_{\mathrm{s}}} \hat{\vec{b}}_{12}^{(i)}\left(t_{n-j}\right)\right) .
$$

A stable float solution reduces the likelihood of large errors in the float solution, which is a major advantage over any covariance-only based triggering.

\section{- Candidate search:}

The LAMBDA method [1] determines integer candidates in a sequential tree search. In the state of the art approach of [1], the candidates are determined at a certain epoch using the respective float solution, which has some-how converged. The fixing is then performed based on these candidates and fully relies on the eventually erroneous float ambiguity covariance matrix.

As the covariance matrix might be erroneous, we determine sets of integer candidates at multiple epochs using different float solutions to increase the likelihood of including the correct candidate in the set of all candidates.

The sets of candidate vectors are merged, i.e. redundant candidates are eliminated and all previous candidates are transformed in case of changing reference satellites. The obtained set of merged candidates are stacked in the pool

$$
N_{\text {pool }}=\left\{N_{12}^{(1)}, \ldots, N_{12}^{(c)}\right\} .
$$

Note that the lengths of the candidate vectors might vary according to the number of tracked satellites at the selected epoch of the float solution. The consideration of the float solution at multiple epochs is of essential importance as the subsequent candidate selection can only be successful if the correct candidate is included in the set. The probability of correct fixing is written as

$$
P_{\text {cf }}=P_{\text {candidate in set }} \cdot P_{\text {correct candidate selected }} \text {. }
$$

\section{- Candidate tracking:}

The LAMBDA method [1] performs a fixing decision using the float solution at a certain epoch. The fixing is trusted if the ratio between the second-lowest and lowest sum of squared ambiguity residuals exceeds a certain value. We have analyzed numerous datasets and have observed that an erroneous candidate is often selected as both the ambiguity residuals and measurement residuals are not trustworthy. There is a strong need for enhancing the fixing decision. One option is to track the candidate vectors over time and to determine a phase-only least-squares baseline estimate at every epoch for every candidate, and to accumulate the sum of squared residuals over time, i.e.

$$
\sum_{j \in j_{s}}\left\|r_{\varphi_{12}}^{(m)}\left(t_{j}\right)\right\|^{2} \forall m
$$

with

$$
\begin{aligned}
r_{\varphi_{12}}^{(m)}\left(t_{j}\right) & =\lambda\left(\varphi_{12}\left(t_{j}\right)-\check{N}_{12}^{(m)}\right)-H\left(t_{j}\right) \check{\vec{b}}_{12}^{(m)}\left(t_{j}\right) \\
& =P_{H}^{\perp}\left(t_{j}\right) \lambda\left(\varphi_{12}\left(t_{j}\right)-\check{N}_{12}^{(m)}\right)
\end{aligned}
$$

with $P_{H}^{\perp}$ being the projector on the orthogonal complement of the space of $H$. The analysis of numerous datasets has shown a certain improvement but a reliable fixing can still not be guaranteed. Therefore, we additionally analyze the error of the least-squares baseline estimate for the $m$-th candidate at epoch $t_{j}$ :

$$
\Delta \check{\vec{b}}_{12}^{(m)}\left(t_{j}\right)=S\left(t_{j}\right) \lambda\left(\Delta \varphi_{\mathrm{MP}_{12}}\left(t_{j}\right)-\Delta \check{N}_{12}^{(m)}\right),
$$

with

$S\left(t_{j}\right)=\left(H^{\mathrm{T}}\left(t_{j}\right) \Sigma^{-1}\left(t_{j}\right) H\left(t_{j}\right)\right)^{-1} H^{\mathrm{T}}\left(t_{j}\right) \Sigma^{-1}\left(t_{j}\right)$,

and $\Delta \check{N}_{12}^{(m)}$ being the error of the $m$-th integer candidate. The fixed phase residuals are shifted by

$$
\Delta r_{\varphi_{12}}^{(m)}\left(t_{j}\right)=P_{H}^{\perp}\left(t_{j}\right) \lambda\left(\Delta \varphi_{\mathrm{MP}_{12}}\left(t_{j}\right)-\Delta \check{N}_{12}^{(m)}\right)
$$


As the $H$ matrix is continuously changing over time due to the satellite movement, erroneous candidates can be detected from the baseline drift or residual drift. The baseline drift is obtained from Eq. (19) as

$$
\begin{aligned}
\check{\vec{b}}_{12}^{(m)}\left(t_{j}\right)-\check{\vec{b}}_{12}^{(m)}\left(t_{1}\right) \\
=S\left(t_{j}\right)\left(\lambda\left(\Delta \varphi_{\mathrm{MP}_{12}}\left(t_{j}\right)-\Delta \check{N}_{12}^{(m)}\right)+\varepsilon_{12}\left(t_{j}\right)\right) \\
\quad-S\left(t_{1}\right)\left(\lambda\left(\Delta \varphi_{\mathrm{MP}_{12}}\left(t_{1}\right)-\Delta \check{N}_{12}^{(m)}\right)+\varepsilon_{12}\left(t_{1}\right)\right) \\
=S\left(t_{j}\right) \lambda \Delta \varphi_{\mathrm{MP}_{12}}\left(t_{j}\right)-S\left(t_{1}\right) \lambda \Delta \varphi_{\mathrm{MP}_{12}}\left(t_{1}\right) \\
\quad+\left(S\left(t_{j}\right)-S\left(t_{1}\right)\right) \Delta \check{N}_{12}^{(m)} \\
\quad+S\left(t_{j}\right) \varepsilon_{12}\left(t_{j}\right)-S\left(t_{1}\right) \varepsilon_{12}\left(t_{1}\right) .
\end{aligned}
$$

Unfortunately, the drift due to the change of $H$ is rather small and compensated to some extent by the change of the weighting $\Sigma^{-1}$ and by the change of the phase multipath over time. However, the drift due to an erroneous integer candidate continuously grows over time. Therefore, the drift is estimated from the baseline estimates of numerous epochs, which are modeled as a linear function of time, i.e.

$$
\begin{gathered}
\left(\begin{array}{c}
\check{\vec{b}}_{12}^{(m)}\left(t_{1}\right)-\check{\vec{b}}_{12}^{(m)}\left(t_{1}\right) \\
\check{\vec{b}}_{12}^{(m)}\left(t_{2}\right)-\overrightarrow{\vec{b}}_{12}^{(m)}\left(t_{1}\right) \\
\vdots \\
\check{\vec{b}}_{12}^{(m)}\left(t_{j}\right)-\check{\vec{b}}_{12}^{(m)}\left(t_{1}\right)
\end{array}\right) \\
=\Lambda\left(\begin{array}{c}
\alpha_{0}^{(m)} \\
\alpha_{1}^{(m)}
\end{array}\right)+\left(\begin{array}{c}
\eta_{12}^{(m)}\left(t_{1}\right) \\
\eta_{12}^{(m)}\left(t_{2}\right) \\
\vdots \\
\eta_{12}^{(m)}\left(t_{j}\right)
\end{array}\right),
\end{gathered}
$$

with $\alpha_{0}^{(m)}$ and $\alpha_{1}^{(m)}$ being the linear coefficients and $\eta_{12}^{(m)}\left(t_{j}\right)$ including the difference of the projected phase noise and multipath, and

$$
\Lambda=\left(\begin{array}{cc}
1 & t_{1}-t_{1} \\
1 & t_{2}-t_{1} \\
\vdots & \vdots \\
1 & t_{j}-t_{1}
\end{array}\right) .
$$

The coefficients are obtained by a standard leastsquares estimation:

$$
\left(\begin{array}{c}
\hat{\alpha}_{0}^{(m)} \\
\hat{\alpha}_{1}^{(m)}
\end{array}\right)=\left(\Lambda^{\mathrm{T}} \Lambda\right)^{-1} \Lambda^{\mathrm{T}}\left(\begin{array}{c}
\check{\vec{b}}_{12}^{(m)}\left(t_{1}\right)-\check{\vec{b}}_{12}^{(m)}\left(t_{1}\right) \\
\check{\vec{b}}_{12}^{(m)}\left(t_{2}\right)-\breve{\vec{b}}_{12}^{(m)}\left(t_{1}\right) \\
\vdots \\
\check{\vec{b}}_{12}^{(m)}\left(t_{j}\right)-\check{\vec{b}}_{12}^{(m)}\left(t_{1}\right)
\end{array}\right) .
$$

The coefficient $\alpha_{1}^{(m)}$ describes the slope/ drift of the baseline due to the erroneous candidate and to some extent due to phase multipath and noise.
- Candidate selection:

The LAMBDA method [1] selects the candidate solely based on the sum of squared ambiguity residuals. This makes the fixing very sensitive to any error in the float solution.

We perform a pre-selection of integer candidates based on the accumulated sum of fixed phase measurement residuals of Eq. (17), i.e. by selecting only the candidates where the accumulated sum of residuals is below a predefined threshold $\gamma$. The candidate with the maximum baseline stability is then chosen among all preselected candidates, i.e.

$\check{N}_{12}^{(m)}=\arg \min _{m}\left|\hat{\alpha}_{1}^{(m)}\right|$ s.t $\sum_{j \in j_{s}}\left\|r_{\varphi_{12}}^{(m)}\left(t_{j}\right)\right\|^{2} \stackrel{!}{<} \gamma$

The joint consideration of the residuals and the baseline stability results in a much more reliable fixing.

An erroneous candidate might still be selected if the integer term in Eq. (22) compensates for the multipath and noise terms, i.e. an erroneous candidate has a lower drift than the correct candidate.

The change of the phase multipath over time is in general not as continuous as the change of the $H$ matrix over time. Thus, the presence of phase multipath could be detected by analyzing the residuals of the drift estimation, i.e.

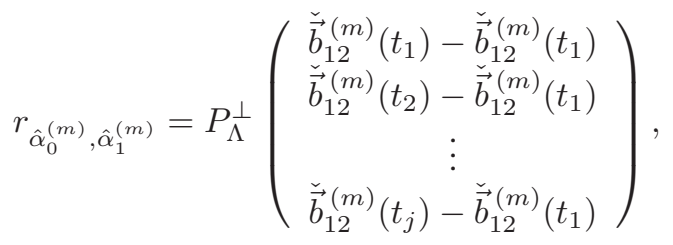

with $P_{\Lambda}^{\perp}$ being the projector on the orthogonal complement of the space of $\Lambda$. As the least-squares baseline estimates $\vec{b}_{12}^{(m)}\left(t_{j}\right)$ are dependent on $\Sigma\left(t_{j}\right)$ and any variation of $\Sigma\left(t_{j}\right)$ might lead to a variation of $\breve{\vec{b}}_{12}^{(m)}\left(t_{j}\right)$, it is recommended to use of constant $\Sigma \mathrm{du}$ ring the candidate tracking and selection phases.

\section{MEASUREMENT RESULTS}

This section includes the verification of our RTK positioning with multipath estimation using real measurements.

We first consider static measurements with an RTK baseline length of $104 \mathrm{~m}$. Fig. 2 shows the code multipath for each double difference over time. One can observe that the change of the code multipath between two successive epochs is for most epochs much lower than the absolute value of the code multipath. This high temporal correlation of the multipath is typical for static environments and is fully exploited in the Kalman filter. The enlarged sections show that the estimated code multipath closely follows the true 
multipath + noise (obtained by subtracting the correct baseline from the DD pseudoranges). The deviation between the estimated multipath and the true multipath + noise is only in the order of magnitude of the code noise.

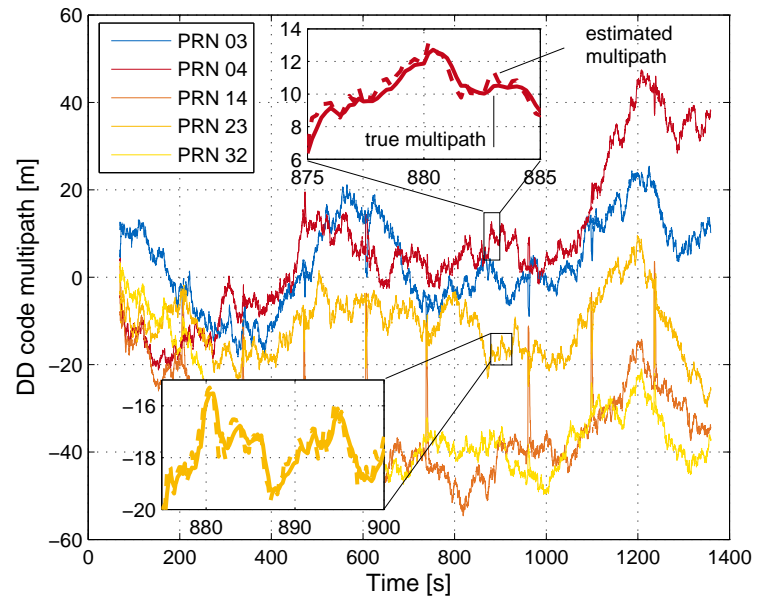

Fig. 2: Estimation of DD code multipath: The true and estimated multipath are shown for all visible satellites. The enlarged sections for PRN 04 and 14 show that the estimated code multipath estimates closely follow the track of the true ones. The error of the multipath estimates are only in the order of magnitude of the code noise.

Fig. 3 shows the accuracy of the float baseline estimate. We can observe that the baseline error reduces to less than $0.5 \mathrm{~m}$ within a few seconds, and then varies by only a few decimeters. The sudden increases in uncertainty every $\sim 200 \mathrm{~s}$ are caused by restarts of the Kalman filter after each ambiguity fixing. If the code multipath is not estimated, the covariance matrix of the code noise has to be increased dramatically to absorb the code multipath in the code noise. The enlarged code noise covariance matrix would result in much longer convergence times. The small error of the float solution shows that the estimation of the code multipath prevents a mapping of the code multipath into other state parameters. Consequently, the estimation of the code multipath is very beneficial.

Fig. 4 shows the DD phase residuals for the fixed solution over time. The residuals of all DD are less than $2 \mathrm{~cm}$ and almost drift-free, which indicates a consistent measurement model and correct integer ambiguity fixing and cycle slip correction. The slight long-term variations are caused by phase multipath, by a changing satellite geometry and/ or by a change of the weighting of the measurements.

Fig. 5 shows the error of the least-squares estimate of the baseline coordinates using only the carrier phase measurements after ambiguity fixing. A millimetre-level positioning accuracy is achievable with low-cost GPS receivers and patch antennas despite severe code multipath. The interpretation is very similar to the previous figure, i.e. the

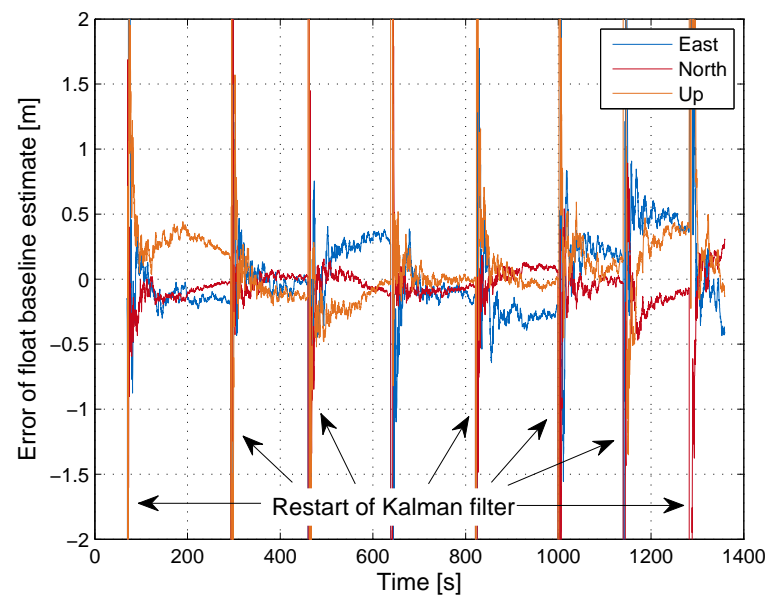

Fig. 3: Accuracy of Kalman filter based float solution with baseline, ambiguity and code multipath estimation: The baseline errors reduce to less than $0.5 \mathrm{~m}$ after convergence of the Kalman filter. This error is in the order of the code noise and much lower than the code multipath. The sudden increases in uncertainty every $\sim 200 \mathrm{~s}$ arise from restarts of the filter after integer ambiguity fixing.

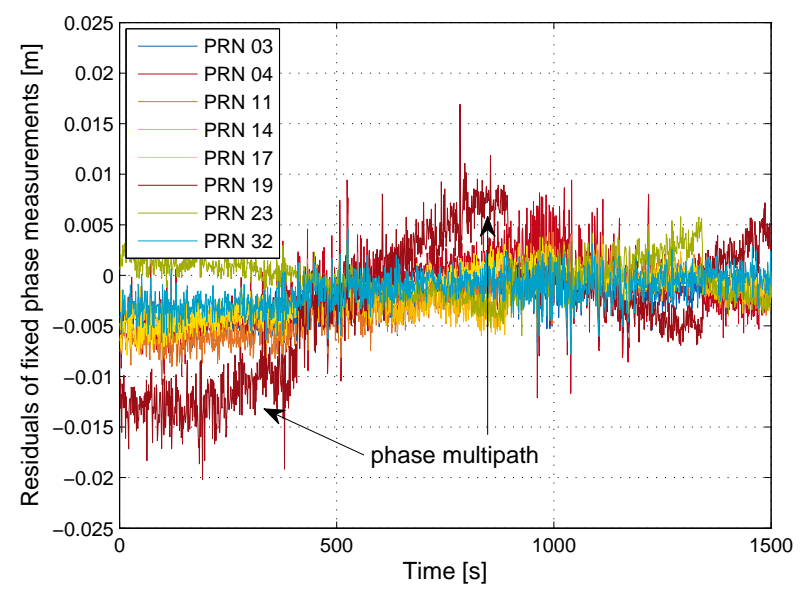

Fig. 4: Residuals of fixed DD carrier phase measurements: The residuals are less than $2 \mathrm{~cm}$, which indicates a correct ambiguity fixing and correct cycle slip correction. The slight long-term variations are caused by phase multipath, by a changing satellite geometry and/ or by a change of the adaptive weighting of the measurements.

small order of magnitude of only $1 \mathrm{~cm}$ indicates a consistent measurement model and a correct ambiguity fixing. The slight long-term variations are due to phase multipath, by a changing satellite geometry and/ or by a change of the weighting of the measurements.

Fig. 6 shows the fixed phase residuals over a period of $\sim 30$ minutes. The low order of magnitude and the lack of a drift indicate a correct integer ambiguity fixing. 


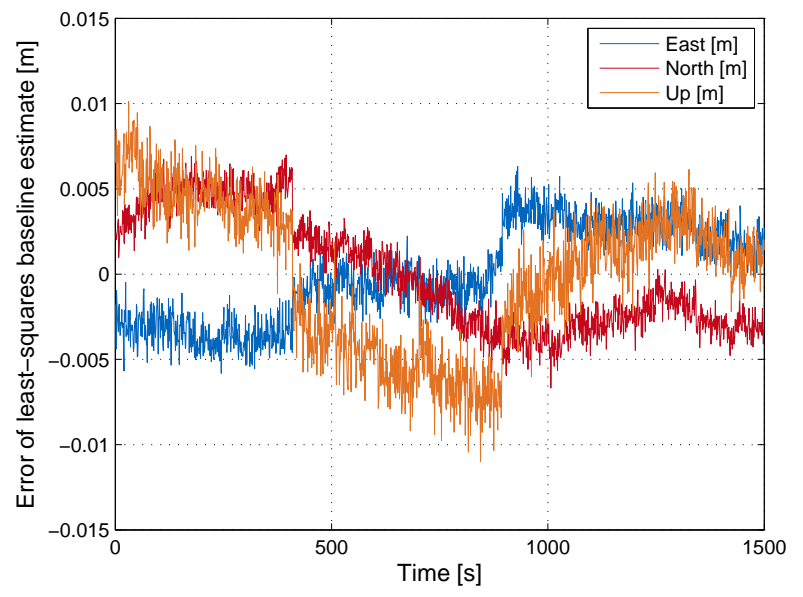

Fig. 5: Error of least-squares ambiguity fixed baseline estimate: A millimetre-level positioning accuracy is achieved with low-cost GPS receivers despite severe code multipath of up to $50 \mathrm{~m}$. The slight temporal variations are caused by phase multipath, by a changing satellite geometry and/ or by a change of the weighting of the measurements.

The time-correlated variations are caused by phase multipath and can be reduced by avaraging the residuals over longer periods.

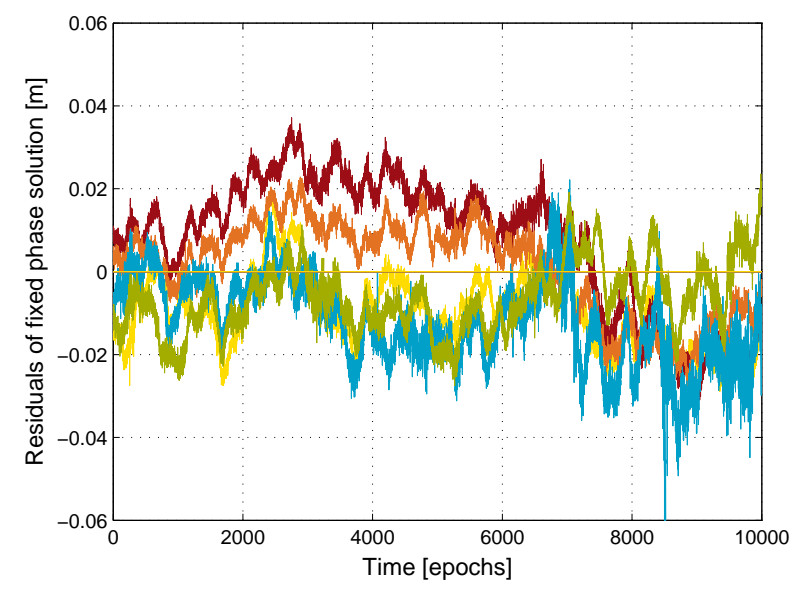

Fig. 6: Long-term variation of fixed phase measurement residuals: the time-correlated variations are caused by phase multipath. The lack of a drift indicates a correct integer ambiguity fixing. The averaging of the residuals over longer periods improves the discrimination between different integer candidates.

Fig. 7 shows the performance of our RTK positioning with GPS/ INS tight coupling in an urban environment: The estimated ambiguity-fixed trajectory closely follows the geodetic reference (Applanix). The deviation between both trajectories is only $9.4 \mathrm{~cm}$ and, thus, below the image resolution of Google Earth.

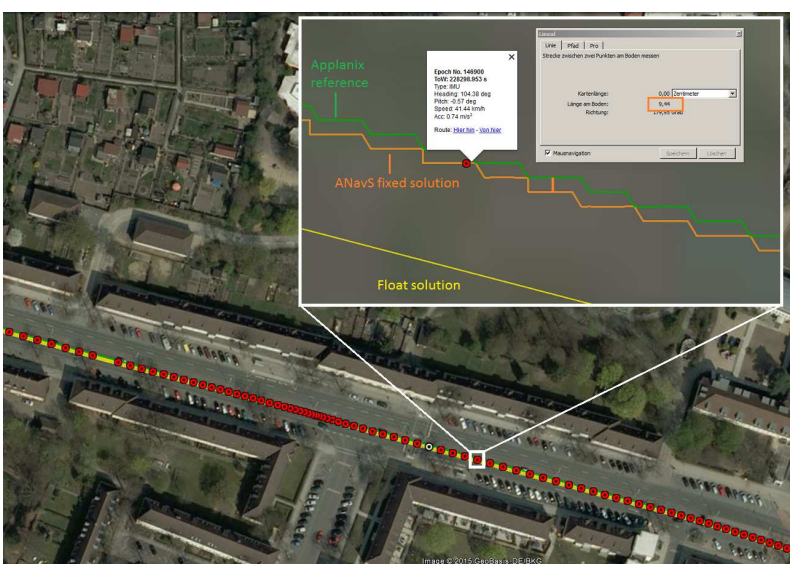

Fig. 7: Performance of RTK positioning with GPS/ INS tight coupling in urban environment: The estimated ambiguity-fixed trajectory closely follows the geodetic reference (Applanix). The deviation between both trajectories is only $9.4 \mathrm{~cm}$ and, thus, below the image resolution of Google Earth.

\section{CONCLUSION}

Multipath is the challenge for RTK positioning with lowcost GPS receivers and antennas. In this paper, we exploited the temporal correlation of the code multipath to enhance the RTK performance. We estimate a code multipath parameter for each double difference in the float solution. The multipath is modeled as a scaled Gauss-Markov process to cover both static and kinematic multipath. The ambiguity fixing includes two phases: an integer collection phase and an integer tracking phase. In the collection phase, we determine candidate vectors at multiple epochs to increase the likelihood of including the correct candidate vector in the set of candidate vectors. In the tracking phase, we determine a single epoch phase-only least-squares fixed baseline estimate for each candidate at each epoch. The residuals are accumulated over time and the candidate vector is selected based on the minimum baseline drift to enhance the reliability of the candidate selection. We applied the proposed method to real measurements of two low-cost GPS receivers and patch antennas, and obtained a millimetre-level positioning accuracy despite code multipath of up to $50 \mathrm{~m}$.

\section{REFERENCES}

[1] Peter J.G. Teunissen, The least-squares ambiguity decorrelation adjustment: a method for fast GPS integer ambiguity estimation, Journal of Geodesy, pp. 65-82, vol. 70, 1995.

[2] Michele Iafrancesco, GPS/ INS tightly coupled position and attitude determination with low-cost sensors, 
Master thesis, Technische Universität München, Sep. 2015.

[3] Herbert Landau, Ulrich Vollath and Xiaoming Chen, Virtual Reference Station Systems, Journal of Global Positioning Systems (CGPS), pp. 137-143, 2002.

[4] Robert G. Brown and Patrick Y.C. Hwang, Introduction to Random Signals and Applied Kalman Filtering with Matlab Exercises, 4th edition, Wiley, 2012.

[5] Andreas Sperl, Joint RTK and Attitude determination, Master thesis, Technische Universität München, Sep. 2015.

[6] C. Jekeli, Inertial navigation systems with geodetic applications, Walter de Gruyter, 1993.

[7] P. Henkel, Tightly coupled Precise Point Positioning and Attitude Determination, IEEE Transactions on Aerospace and Electronic Systems (TAES), accepted, 2015.

[8] Patrick S. Henkel and Michele Iafrancesco, Tightly coupled Position and Attitude Determination with two low-cost GNSS receivers, Proc. of 11-th Intern. IEEE Symp. on Wireless Communication Systems (ISWCS), Barcelona, Spain, pp. 895-900, Aug. 2014. 